\title{
5G Evolution for Multicast and Broadcast Services in 3GPP Release 17
}

\author{
Vinay Kumar Shrivastava, Sangkyu Baek and Youngkyo Baek
}

\begin{abstract}
G communications system is on the evolution path with present focus on enabling advanced features and new service capabilities. Multicast and Broadcast Services (MBS) are being considered as one of the most promising use cases of 5G. As a late entrant to 3rd Generation Partnership Project (3GPP) 5G standards, MBS work item is presently being developed over existing 5G framework, and is targeted to enhance 5G New Radio and 5G Core Network capabilities for a reliable, low latency, resource efficient, and massive deployment of a wide array of multicast and broadcast services. MBS development entails enhancements for network and user equipment side on architectural, protocol and transmission aspects in order to address a new set of multicast and broadcast service requirements in 5G. In this article, we present an introduction to MBS standardization and outline newly introduced technical features, and their use cases.
\end{abstract}

Index Terms-MBS, 5G, NR, multicast, broadcast.

\section{INTRODUCTION}

$\mathbf{M}$ ulticast and broadcast communications have been considered to pave the way for resource efficient transmission to multiple end users which require to receive same contents. Due to this obvious gain, 3GPP's Long Term Evolution (LTE) standards have introduced various features to support the broadcast services over more than a decade. Historically, evolved Multimedia Broadcast and Multicast Service (eMBMS) [1][2] in LTE Release 9-12 was an initial set of standardized features directed towards this objective.

eMBMS is based on Single Frequency Network (SFN), and it utilizes synchronized multi-cell transmissions from many eNBs (evolved NodeBs i.e. base stations in 3GPP network) together providing for over-the-air combining of MulticastBroadcast SFN (MBSFN) signals, enhancing the reliability and coverage areas for services. MBSFN transmissions are time-interleaved with unicast transmission with pre-assigned and dedicated sub-frames over the radio frame and utilize full system bandwidth.

To overcome the limitations of eMBMS, Single Cell PointTo-Multipoint (SC-PTM) was motivated and introduced in Release 13 [3][4]. SC-PTM adopted a more flexible approach where dynamic time and frequency resource utilization (even within a sub-frame) is possible for broadcast services over a small dense geographical region (e.g. hotspot) within a singlecell coverage. The dynamic resource utilization effectively allows integration of broadcast services delivery with unicast physical channels.

Vinay Kumar Shrivastava is with Samsung Research Institute, Bengaluru, India, e-mail: (shrivastava@ samsung.com).

Sangkyu Baek and Youngkyo Baek are with Samsung Research, Seoul, Korea, e-mail: (sangkyu.baek@samsung.com, youngkyo.baek@samsung.com).
Release 14/15 targeted broadcast services with dedicated carrier for MBMS transmission supporting up to $100 \%$ resource usage for MBMS and a large duration Orthogonal Frequency Division Multiplexing (OFDM) symbol to support wider coverage in order of tens of kilometres. Release 16 introduced 'Terrestrial Broadcast' targeting Enhanced Television (EN-TV) services over large and static transmission areas with dedicated broadcast infrastructure, for example, HighPower High-Tower (HPHT) deployments [5]. Despite several enhancements across different releases, LTE eMBMS and SCPTM lacked on reliability and latency aspects for multicast and broadcast services with no mechanism in place for error correction and packet retransmission at radio interface and protocol layers.

Meanwhile, Fifth Generation Wireless Communications Technology (5G) has been standardized by $3 \mathrm{GPP}$ and commercially launched in 2019. However, 5G did not address multicast and broadcast service in its first phase (Release 15) [6] and second phase (Release 16) [7] packages, with initial focus being on enhanced Mobile Broadband (eMBB) and Ultra-Reliable and Low-Latency Communication (URLLC). Consequently, as a late entrant in 5G Release 17,3GPP started to build functional support of multicast and broadcast services abbreviated as MBS over an existing 5G standards framework. The standardization has been conducted for overall $5 \mathrm{G}$ system architecture from both Next Generation Radio Access Network (NG-RAN) and 5G Core Network (5GC) perspectives.

Although a natural adoption and evolution of multicast and broadcast services in 5G standards was expected, it also posed new requirements and challenges for 5G MBS. New emerging multicast services in MBS such as mission-critical delaysensitive signaling and high-resolution IPTV require to achieve same levels of high reliability and low latency as available with unicast services and therefore, demand for an involved protocol stack design with layers and functionalities reinforcing on the reliability and latency aspects in RAN protocol, physical layer and service continuity. On contrary, broadcast services in MBS can maintain broadly same requirements like LTE eMBMS and SC-PTM, and therefore, inherit many design features.

Also, unique 5G New Radio (NR) characteristics such as BandWidth Part (BWP), beamforming, absence of always-ON reference signals (i.e. Cell-specific Reference Signal, CRS, in LTE), varied Sub-Carrier Spacing (SCS), non-SFN deployments and new transmission aspects significantly influence NR MBS design. In 5GC, wider-area service with using not only new MBS functions but also existing network function is further required. 

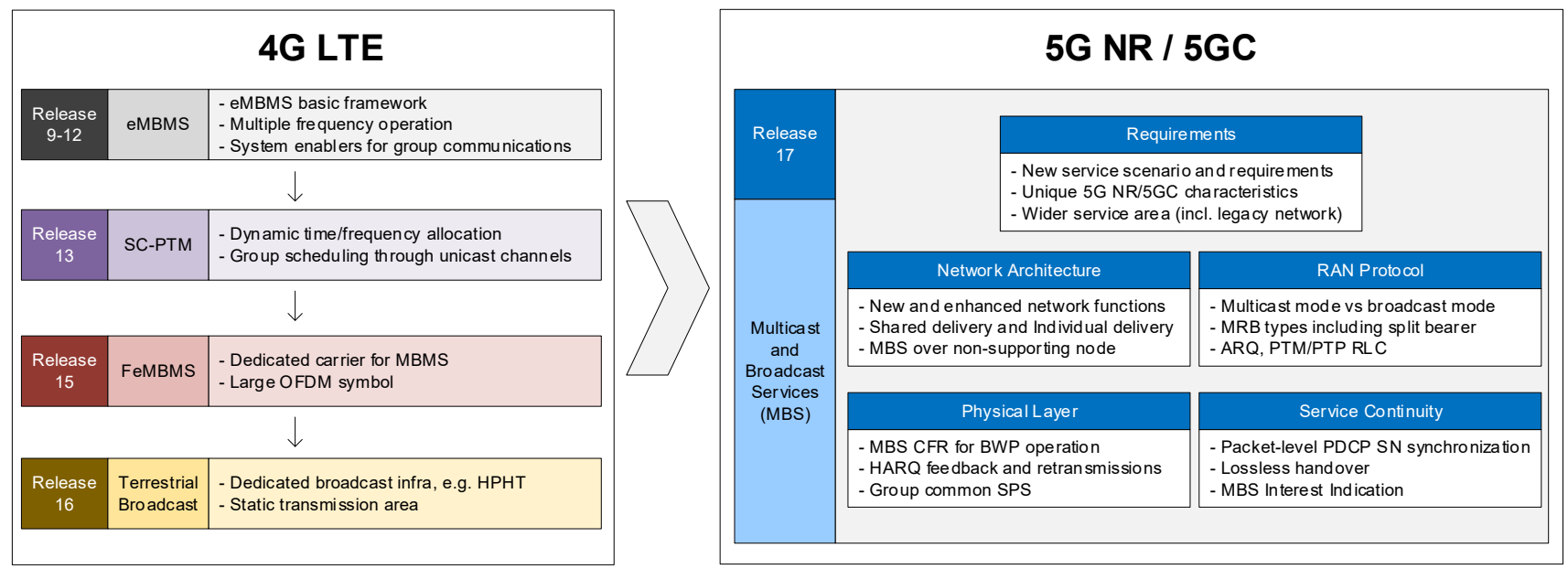

Fig. 1: Multicast/broadcast services evolution for LTE and 5G MBS and certain key technical aspects.

In this regard, key features of the standardized 5G MBS are as follows:

- Group scheduling mechanism to allow UEs to receive MBS service including simultaneous operation with unicast reception

- Multicast delivery in 5GC, i.e. shared delivery

- Reliability enhancements by dynamic change of multicast/broadcast service delivery between Point-toMultipoint (PTM) and Point-to-Point (PTP), Automatic Repeat Request (ARQ), and Hybrid Automatic Repeat Request (HARQ), etc.

- Supporting mobility and lossless handover

- Reception of broadcast data irrespective of UE's Radio Resource Control (RRC) states

- MBS over legacy network node, for example, Release 15/16 network.

At present, 5G MBS discussion in 3GPP Working Groups (WGs) is progressing with a targeted completion of functional specifications by March 2022. Figure 1 depicts the evolution of 5G MBS from legacy LTE and involved aspects. The reminder of this article provides comprehensive overview of each aspect of 5G MBS from service requirements to functional enhancements.

\section{NeW SERVICE REQUiREMENTS}

MBS is targeted to enable diverse services including public safety and mission critical, Vehicle-to-Everything (V2X) applications, transparent IPv4/IPv6 multicast delivery, IPTV, software delivery over wireless, group communications and IoT applications. Service requirements for different uses cases of MBS differ in their requirements for reliability, latency, QoS handling, service area coverage, service continuity and security aspects. Therefore, it becomes imperative to build a comprehensive mechanism for MBS to address these diverse needs.

Reliability and latency: For MBS, high reliability and lowlatency services (e.g. mission critical delay-sensitive signaling with packet delay budget of $60 \mathrm{~ms}$ and packet error rate of $10^{-6}$ ) present extreme service requirements as shown in Table I [8].
Therefore, there is a new need for dynamic adaptation of service delivery modes to meet QoS constraints and approaches for UE providing feedback and availing retransmission to ensure reception reliability. Application layer Forward Error Correction (FEC) in LTE eMBMS/SC-PTM requires some amount of latency to be effective, that is, latency should be multiple times the coherence times of the channel [9]. Consequently, NR MBS has a new requirement to consider physical layer FEC to support low latency services, albeit at the cost of increased complexity at physical layer.

QoS handling: MBS service may have multiple streams which may differ in their QoS requirements and need to be treated differentially. These streams can be mapped to appropriate QoS flows as per their requirements, and to appropriate radio bearers or logical channels at the radio protocol level for enforcement of the QoS requirements.

Service area coverage: Different services have varied requirements in terms of service area coverage. For example, V2X application has shorter coverage needs than IPTV services. MBS has no standardized support for SFN, and service transmission area is expected to be limited to a single cell or an area of multiple cells based on network configuration to meet different service requirements.

Service continuity: When a UE is moving across geographic regions, UE's serving cell can be changed (i.e. handover), and non-negligible latency due to the cell change may occur. However, longer latency exceeding the packet delay budget degrades the quality of the user's experience, thus it should be minimized. Special provisions are inevitable to ensure packetlevel synchronization and data forwarding across different network nodes, and also considerations for different delivery modes of the UE across handover. As MBS coverage may not be ubiquitous, especially during initial deployments, service continuity across legacy network nodes is an essential requirement and may involve transition in service delivery modes (e.g. unicast based access for MBS services).

Security: To ensure secure communication for certain MBS services (e.g. software delivery, mission critical service), a security functionality is required which provides for session 
TABLE I: Service QoS requirements for different use cases of MBS.

\begin{tabular}{|l|c|c|c|c|}
\hline Service & Resource Type & Default Priority Level & Packet Delay Budget & Packet Error Rate \\
\hline Mission-critical Push-To-Talk & GBR & 7 & $75 \mathrm{~ms}$ & $10^{-2}$ \\
\hline Mission-critical delay-sensitive signaling & Non-GBR & 5 & $60 \mathrm{~ms}$ & $10^{-6}$ \\
\hline IPTV & Non-GBR & 70 & $100 \mathrm{~ms}$ & $10^{-3}$ \\
\hline V2X messages & Non-GBR & 65 & $50 \mathrm{~ms}$ & $10^{-2}$ \\
\hline
\end{tabular}

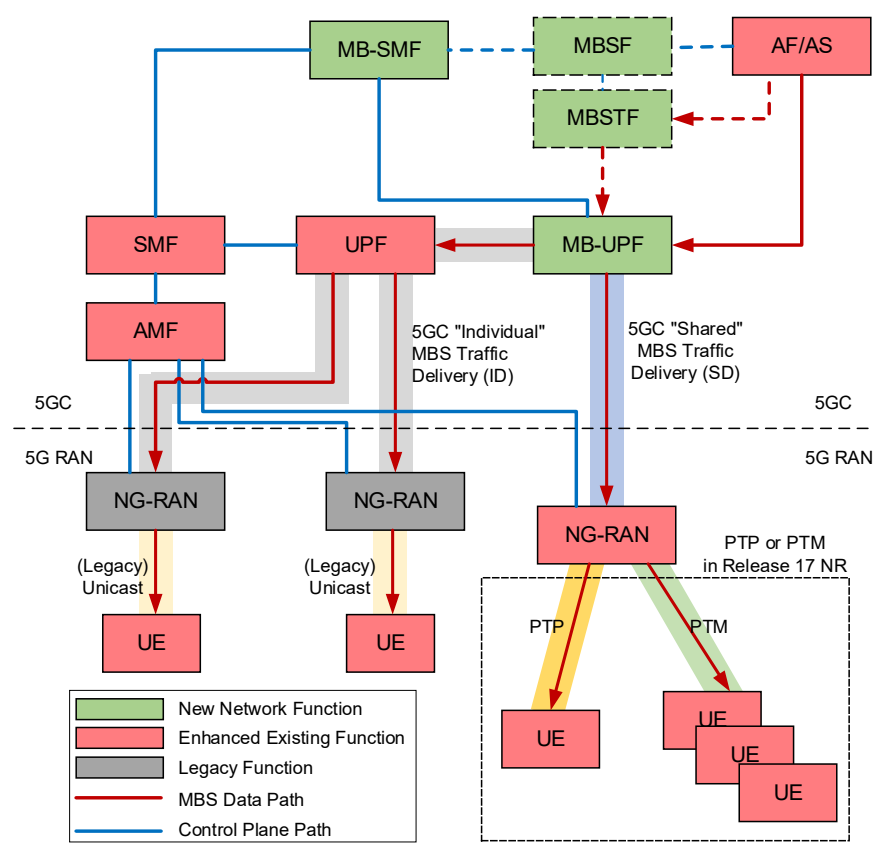

Fig. 2: 5G MBS system architecture and user plane data transmission.

security context, access credentials and their maintenance across involved network nodes and UEs. In particular, ciphering and integrity protection are required similar to the unicast transmission. Security requirements can be fulfilled with two potential realizations for 5G MBS, namely, (a) NG-RAN based security, which involves Protocol Data Convergence Protocol (PDCP) layer based mechanism, and (b) application/service layer security provisioning, in which case it becomes transparent to NG-RAN and radio protocols.

\section{Network ARChitecture}

The network architecture of $5 \mathrm{G}$ system (5GS) is enhanced to support multicast and broadcast services, with the design aimed to re-use legacy $5 \mathrm{G}$ system as much as possible. The multicast is defined as the same service and the same specific content data that are provided simultaneously to a dedicated set of UEs which are authorized in the service coverage. The broadcast is defined as the same service and the same specific content data that are provided simultaneously to all UEs in the service area. Which cast type is actually used depends on actual service type.

To support MBS in 5GS, some network functions are newly introduced as depicted in Fig. 2.

- Multicast Broadcast User Plane Function (MB-UPF) is an ingress point to $5 \mathrm{GS}$ and works as a session anchor to $5 \mathrm{GS}$.
- Multicast Broadcast Session Management Function (MBSMF) manages MBS session and configures a user plane function MB-UPF, based on the policy rules for multicast and broadcast services.

- Multicast Broadcast Service Function (MBSF) has service level functionality to interact with AF/AS (Application Function/Application Server) and MB-SMF for MBS session operations. Further, it determines transport parameters and session transport, and control MBSTF if used, which can be implemented in the Network Exposure Function (NEF).

- Multicast Broadcast Service Transport Function (MBSTF) has generic packet transport functionalities available to any IP multicast enabled application such as framing, multiple flows, packet FEC encoding, and therefore, works as a media anchor for MBS data traffic [10].

Moreover, existing network functions are enhanced for MBS. For example, Session Management Function (SMF) is enhanced to handle UE's multicast session join or leave and to determine the MBS traffic delivery method to use. Access and Mobility Management Function (AMF) is enriched to select appropriate NG-RAN nodes for broadcast and group notification of multicast session activation towards UEs. User Plane Function (UPF) is enhanced to deliver multicast data to UEs via PDU session. Policy Control Function (PCF) is upgraded to support QoS handling for MBS session.

On top of the enhanced network architecture, two delivery methods are supported to deliver the MBS data traffic from MB-UPF to NG-RAN, namely, 5GC Shared Delivery (SD) method and 5GC Individual Delivery (ID) method.

SD is used to save $5 \mathrm{GC}$ resource for MBS data transmission. More specifically, when an MB-UPF has a packet to transmit to multiple UEs receiving a data of MBS session, it just sends a single copy of MBS data packet to each NG-RAN node. This single packet is not dedicated to a single UE but ultimately shared by multiple UEs. Obviously, only NG-RAN nodes with MBS capability can receive data by SD whereas NG-RAN node which does not support MBS capability, for example a legacy gNB, cannot receive data by SD. SD can be used for both multicast and broadcast services.

In ID, when an MB-UPF has a packet to transmit to multiple UEs receiving data of MBS session, a single copy of MBS data packet is delivered to the UPF and which, in turn, sends separate copies corresponding to individual UEs. Although ID seems to be less efficient from resource consumption perspective, it is essential for cases wherein NG-RAN node does not have MBS capability of receiving data by SD but data transmission of MBS session is still required. For instance, in a mobility scenario, a UE which is receiving MBS data packets, can move to other gNB's coverage not supporting 

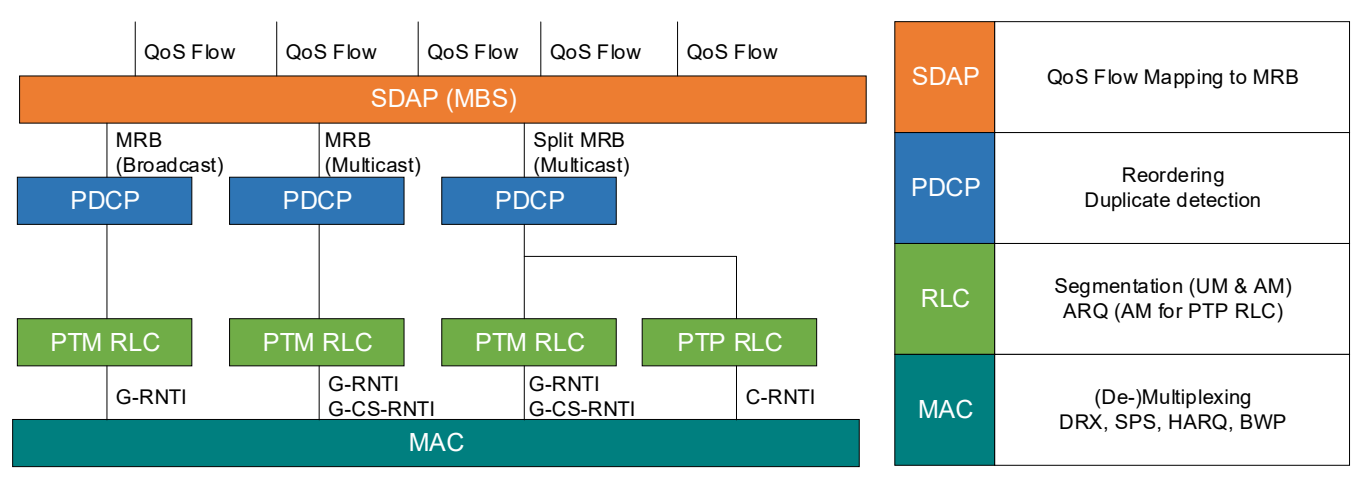

Fig. 3: Protocol architecture for NR MBS.

MBS capability; network switches its delivery method to ID for service continuity. Since ID is destined to a particular UE, it is applied to multicast service only.

When the content provider or AF wants to disseminate MBS services, it may request the 5GS to configure the MBS session via NEF (or MBSF). Information for MBS service reception can be provided to UE via service announcement from AF. Such information consists of service type, MBS Session ID, IP multicast address and so on.

For multicast service reception, UE joins an associated MBS session identified by the MBS Session ID. Thereafter, transmission resources are established for the multicast data transmission to the serving NG-RAN node and to UE, wherein if the shared delivery method is used, then PTM and/or PTP delivery mode is utilized towards UE(s) on the air interface.

When the data is not received in the MB-UPF, or AF requests for the MBS session, network resource for the MBS session at the NG-RAN node can be deactivated. Further, when the data is received in the MB-UPF, or AF requests for the MBS session, resource for the MBS session at the NG-RAN node(s) can be activated via group notification based on the MBS Session ID called Temporary Mobile Group Identity (TMGI).

\section{Protocol Architecture}

NR MBS supports two delivery modes, namely, multicast mode (delivery mode 1) and broadcast mode (delivery mode 2). Each mode has its own characteristics and target services.

Target services of multicast mode have particular QoS requirements which the network should guarantee as in case of unicast. Therefore, UEs receiving multicast data are required to stay in CONNECTED state and a dedicated RRC signaling provides the radio resource configuration including MRB configuration, physical layer configurations and so on, which can be optimally configured based on interaction between $\mathrm{UE}$ and gNB. Moreover, in case that reliable transmission is required for cell edge user with bad channel quality, the transmission can be switched to PTP leg, that is, PTP RLC and ARQ can enhance the performance as in unicast transmission.

When no multicast data arrival is expected, the multicast session can be deactivated and UEs belonging to the multicast group can transit to INACTIVE or IDLE state. These UEs have to re-enter CONNECTED state when the multicast session is about to be re-activated. In this case, group paging with the corresponding MBS Session ID is used to wake-up these UEs.

On the contrary, broadcast mode can be provided to all UEs within a coverage regardless of RRC states. The broadcast mode is a similar mechanism to SC-PTM in LTE. In order for UEs out of CONNECTED state to receive the broadcast data, radio resource configuration for broadcast mode is periodically transmitted via MBS Control Channel (MCCH) from which UEs apply the received configuration for MBS Traffic Channel (MTCH).

The broadcast mode does not require any interaction between UE and gNB. The network does not have any feedback from UE side on transmission status (e.g. ACK/NACK) but it transmits the data only in the best-effort manner. Hence, QoS cannot be guaranteed and only low-QoS services are feasible. Also, the broadcast mode does not mandate RRC state transition.

Figure 3 describes radio protocol architecture for NR MBS. It is designed to reuse existing functionalities of unicast, including four sub-layers, namely, Service Data Adaptation Protocol (SDAP), PDCP, RLC, and Medium Access Control (MAC) [11].

SDAP has a one-to-one mapping with MBS session serving multiple QoS flows (QFs) received from 5GC. SDAP allocates MBS Radio Bearer (MRB) transmitted for QFs based on required QoS and the network's policy. Since MBS is only for downlink transmission, uplink-related SDAP functions, for example, reflective QoS and network-initiated QF remapping, are not supported.

PDCP is used to support reordering function under an assumption that a UE may receive packets out of order due to ARQ or HARQ retransmissions of the lower layers. A PDCP entity can be linked to multiple RLC entities for PTM-PTP switching where gNB may send packets via either PTP RLC, PTM RLC or both. If the gNB sends the same sequenced PDCP packet via both RLCs, duplicate detection function in PDCP discards the later arrived one.

RLC is responsible for segmentation and ARQ [12]. gNB's segmentation function fragments a large-sized packet into several small-sized sub-packets by considering available resource size. UE's RLC entity reassembles the original packet and delivers to upper layer (i.e. PDCP).

There are two types of RLC entities, namely, PTM RLC 


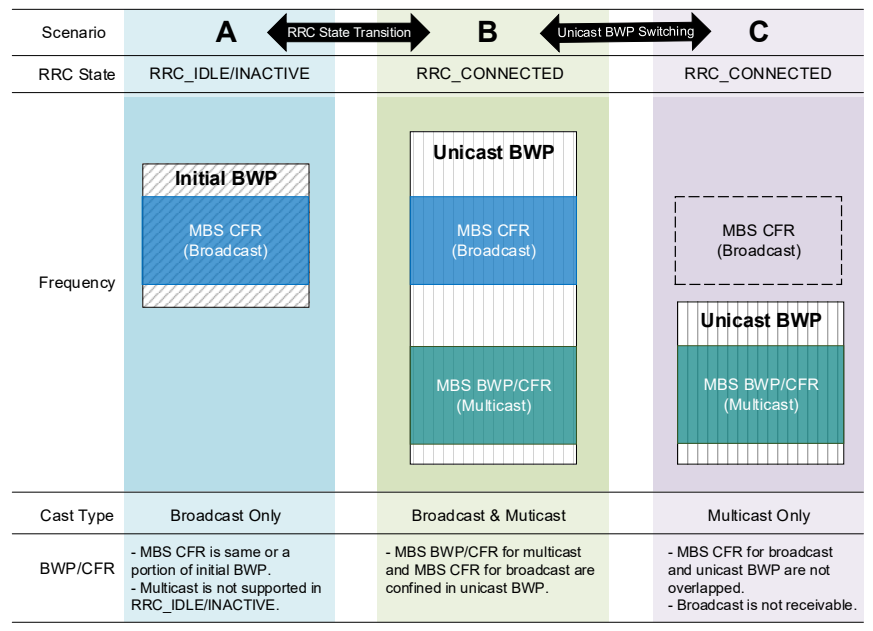

Fig. 4: An example for BWP/CFR operation and usage for MBS broadcast and multicast reception.

and PTP RLC. PTM RLC is a group-common RLC where all UEs configured with this RLC can receive the same packets by using shared Group Radio Network Temporary Identifier (G-RNTI) or Group Configured Scheduling Radio Network Temporary Identifier (G-CS-RNTI). Due to complexity of synchronization of PTM RLC entities at multiple UEs and gNB, PTM RLC does not support Acknowledged Mode (AM) but support Un-acknowledged Mode (UM). PTP RLC is a UEdedicated RLC where the packet is transmitted only to a particular UE using Cell Radio Network Temporary Identifier (CRNTI) or CS-RNTI. Instead, PTP RLC enables $100 \%$ reliable transmission by supporting ARQ (i.e. RLC AM). gNB would select an RLC entity (or both RLC entities) appropriately to transmit packets by considering required reliability, UE's link quality, cell congestion status, and so on.

MAC supports multiplexing and de-multiplexing of data from/to multiple RLC entities. For UE power saving, MBS session specific Discontinuous Reception (DRX) can be configured for a UE receiving MBS data. MAC manages relevant physical layer schemes, for example, BWP, HARQ operation and Semi-Persistent Scheduling (SPS).

\section{Physical Layer Aspects}

One of the most important NR physical layer characteristics influencing MBS design is BWP operation in which UE does not monitor whole frequency bandwidth but monitors only a particular BWP, i.e. active BWP for data scheduling and control information in CONNECTED state and Initial BWP for receiving paging and system information in IDLE/INACTIVE state. Considering the BWP operation, Release 17 NR introduced MBS Common Frequency Resource (CFR) on which multiple UEs can commonly perform MBS reception. For multicast mode supported in only CONNECTED state, Multicast CFR for receiving group-common Physical Downlink Control Channel (PDCCH) and Physical Downlink Shared Channel (PDSCH) is confined within the UE's dedicated active unicast BWP to support simultaneous reception of unicast and multicast in the same time slot. Starting PRB
(Physical Resource Block) and length of PRBs and associated group-common PDCCH and PDSCH configurations specify the Multicast CFR. It is worth noting that SCS and CP (Cyclic Prefix) for CFR are same as that for active unicast BWP in order to avoid BWP switching or additional hardware receiver capability. Further, it is also possible for gNB to schedule unicast transmission on the frequency resources specified by Multicast CFR. The multicast mode is suitably provisioned along with unicast reception with regard to BWP, channel measurement, Channel State Indicator (CSI) reporting. For broadcast reception supported in all RRC states, Broadcast CFR for receiving group-common PDCCH and PDSCH is confined within the Initial BWP. For this approach, Broadcast CFR has the same frequency resources as Control Resource Set index 0 (CORESET0) [13] for receiving group-common $\mathrm{PDCCH}$ and $\mathrm{PDSCH}$, which carries data from $\mathrm{MCCH}$ and MTCH logical channels.

Figure 4 illustrates the BWP operation and usage for MBS. Imperatively, for a UE to receive both multicast and broadcast in CONNECTED state, it is needed that the dedicated unicast BWP not only includes the Multicast CFR but also overlaps with the Broadcast CFR along with same SCS and CP. Since the broadcast reception for CONNECTED state UE is on best effort basis, it is not guaranteed that UE always receives data of broadcast mode in case that Broadcast CFR is located outside of UE's active unicast BWP.

Unlike LTE eMBMS and SC-PTM, HARQ-ACK feedback and HARQ retransmissions are supported to achieve high reliability for multicast mode. HARQ-ACK feedback is required for gNB to know UE's reception status and perform the retransmission. However, feedback resource in PUCCH may be overloaded when many UEs are served for a multicast session. Moreover, a criterion of retransmission could be failure of reception at one UE. Based on these factors, configuration flexibility of HARQ-ACK feedback options is allowed as follows:

- ACK/NACK based HARQ-ACK feedback: UE feedbacks ACK or NACK over a UE dedicated PUCCH resources. This mechanism be efficient when the number of UEs receiving the multicast data is small.

- NACK only based HARQ-ACK feedback: UE feedbacks only NACK over common PUCCH resources shared with other UEs in same group. This mechanism is resourceefficient but gNB cannot detect the case that UE fails decoding of PDCCH information.

- No HARQ-ACK feedback: UE does not send any feedback for received data. When the QoS requirement for the multicast data for UE is low, gNB can use this option to save the PUCCH resource. gNB can dynamically switch between ACK/NACK based HARQ-ACK feedback and No HARQ-ACK feedback by RRC signaling or Downlink Control Information (DCI).

HARQ retransmission has further flexibility to use either PTM-type or PTP-type retransmission, after the initial transmission of PTM-type destined to multiple UEs. PTMtype retransmission is a typical retransmission to the same recipients of the initial transmission by using shared G- 


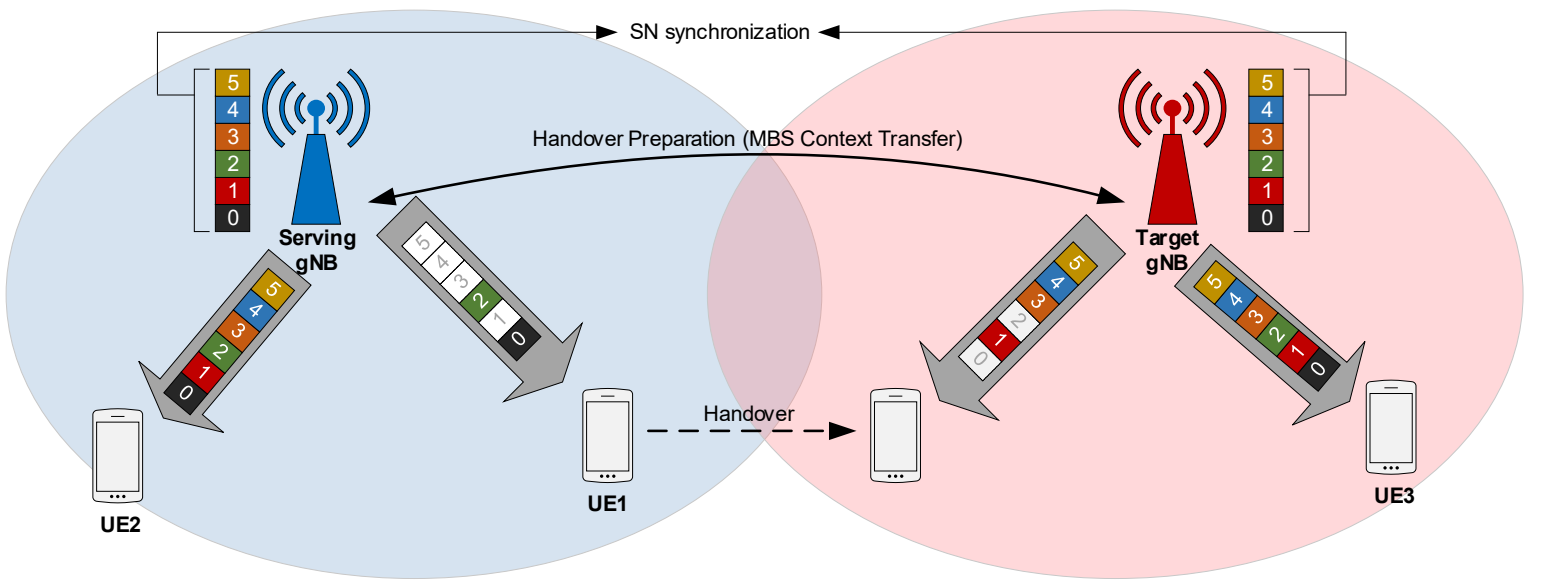

Fig. 5: An illustration for packet-level service continuity with lossless mobility in multicast mode.

RNTI. On the contrary, PTP-type retransmission is a dedicated retransmission for a single UE by using UE's C-RNTI. This PTP-type retransmission would be beneficial when optimal resource configuration such as modulation and coding scheme (MCS) is possible for the retransmission.

Different to LTE eMBMS, which is based on Physical Multicast Channel (PMCH) and common MBSFN Reference Signal (MRS) from multi-cell transmissions, NR MBS uses same UE specific CSI-RS reference signal present on PDSCH for channel measurement and CSI feedback as for NR unicast [14]. Maximum number of time-division multiplexed PDSCH receptions capability in a slot per serving cell is same as for legacy NR Releases. This is shared across for group common PDSCHs for MBS and unicast PDSCHs. In order to provide multiple simultaneous MBS services, UE can receive multiple G-RNTIs or G-CS-RNTIs based transmissions in parallel and each service can correspond to each pair of G-RNTI and G-CS-RNTI. For services with periodic service pattern, e.g. voice/video call, up to eight SPS configurations in a BWP of a serving cell can be simultaneously configured and shared by multiple UEs.

\section{Mobility and Service Continuity}

Figure 5 depicts an example of handover scenario with service continuity and lossless mobility supported in multicast mode. When UE changes its serving cell due to the mobility (e.g. handover), the support of service continuity is important for users not to experience interruption or performance degradation of the ongoing services. The service continuity of $5 \mathrm{G}$ MBS is achieved based on packet-level sequence number (SN) synchronization where the same packet has the same PDCP $\mathrm{SN}$ over the area supporting the service continuity.

In unicast, PDCP SN for a signaling radio bearer (SRB) or data radio bearer (DRB) of a UE can be continued during handover, utilizing handover preparation procedure with indication of the current SN status and data forwarding from serving gNB to target gNB [15]. In multicast, multiple UEs over the large service area would simultaneously receive packets of an MRB. Therefore, the packet-level SN coordination among all gNBs of the service area is required at the beginning of MRB initialization. The SN synchronization naturally enables UEs to maintain the configured MRBs during the handover. The handover preparation requires only context transfer of the UE which is about to handover to the target gNB. Alike unicast, all MBS configurations used after the handover are provided by the target gNB.

Since the connection with the source gNB is released at the handover, the packet loss could occur but this can be recovered through retransmission by the target gNB. This recovery is similar to the unicast handover where the target gNB selectively retransmits packets which are not yet successfully delivered to the UE based on PDCP status report. In other words, service continuity with lossless handover can be eventually supported for multicast.

Handover to gNB not supporting MBS features, for example, involving legacy $\mathrm{gNB}$, is a possible scenario considering backward compatibility. In this case, the individual delivery method is taken instead of the shared delivery method for MBS traffic delivery during the handover so that the serving gNB needs to change the ongoing MRB to unicast DRB in advance for the target node to understand the transferred UE context. In case the target gNB does not establish the moving UE's ongoing MRBs but support MBS, the service continuity with lossless handover can still be supported by the context transfer and establishment of the MRBs.

In broadcast mode, network does not accurately know which UEs are receiving or interested in the broadcast service. Hence, the enhanced service continuity of multicast mode is not reused for broadcast mode, and only low-level service continuity without lossless handover can be possible. $\mathrm{MCCH}$ provides the broadcast configurations possibly including neighbor cell information which UE can directly apply for cell reselection. Also, when the serving gNB does not provide UE's interested broadcast services, UE can send an MBS Interest Indication (MII) message to request UE's interested services which have not been provided. Although these mechanisms have some latency of hundreds of milliseconds, the ongoing broadcast services can be continued with low QoS requirement. 


\section{Conclusions And Future Directions}

In this article, we provided an overview of $5 \mathrm{G}$ MBS, its key technical features and standardization progress in $\mathrm{Re}$ lease 17. Diverse service requirements and evolving network architecture aspects for MBS were discussed. Certain stringent MBS service requirements for high reliability and low latency led to involved radio protocol layers and functions for MBS operation. While NR physical layer aspects and unique transmission characteristics influenced MBS design and new approaches for MBS CFR, HARQ ACK/NACK feedback and retransmission were realized. Lastly, mobility and service continuity provisions to ensure lossless/ seamless MBS service reception were introduced.

It is expected that Release 17 version of MBS may not be able to address many aspects due to lack of time, for example, multicast reception is presently restricted to CONNECTED state UEs only. Further, MBS feature will continue evolving in future releases of $5 \mathrm{G}$ and we recognize there are certain important areas and aspects that would be potential candidates, namely

- Multicast service reception and continuity across IDLE /INACTIVE state to cater large user base and critical services

- MBS for localized services and its integration to private networks

- Energy efficiency for MBS transmission

- Extended service coverage for MBS services

- New service types, including support for EN-TV and Receive-Only-Mode (ROM) services that are excluded from Release 17

- Multi-Radio Dual Connectivity (MR-DC) deployments for MBS

To conclude, MBS is set on an interesting journey of technical progress, standardization and market deployments with $5 \mathrm{G}$.

\section{REFERENCES}

[1] D. Lecompte, and F. Gabin, "Evolved Multimedia Broadcast/Multicast Service (eMBMS) in LTE-Advanced: Overview and Rel-11 Enhancements", IEEE Commun. Mag., vol. 50, no. 11, pp.68-74, Nov. 2012.

[2] J. Kim et al., "Group communication over LTE: A radio access perspective", IEEE Commun. Mag., vol. 54, no. 4, pp. 16-23, Apr. 2016.
[3] Y. Zhang et al., "MBSFN or SC-PTM: How to Efficiently Multicast/Broadcast", IEEE Trans. Broadcasting, Early Access, DOI: 10.1109/TBC.2021.3062551.

[4] 3GPP TR 36.890, Study on single-cell point-to-multipoint transmission for E-UTRA, v13.0.0, July 2015.

[5] J. J. Gimenez et al., "Enhanced TV delivery with EMBMS: Coverage evaluation for roof-top reception", Proc. IEEE Int. Symp. Broadband Multimedia Syst. Broadcast. (BMSB), pp. 1-5, 2018.

[6] S. Parkvall et al., "NR: The New 5G Radio Access Technology", IEEE Commun. Standards Mag., Dec. 2017.

[7] Y. Kim et al., "New Radio (NR) and Its Evolution toward 5G-Advanced", IEEE Wireless Commun., vol. 26, no. 3, pp. 2-7, June 2019.

[8] 3GPP TS 23.501, System architecture for the 5G System (5GS), Stage 2, v17.1.1, June 2021.

[9] 3GPP TR 26.881, Study on Forward Error Correction (FEC) for Mission Critical Services, v15.0.0, Mar. 2018.

[10] 3GPP TS 23.247, Architectural enhancements for 5G multicastbroadcast services, Stage 2, v0.3.0, May 2021.

[11] E. Dahlman, S. Parkvall and J. Sköld, "5G NR - The Next Generation Wireless Access Technology”, Oxford, U.K.:Academic Press, 2018.

[12] 3GPP TS 38.322, Radio Link Control (RLC) protocol specification, v16.2.0, June 2021.

[13] K. Takeda et al., "Understanding the heart of the 5G air interface: An overview of physical downlink control channel for $5 \mathrm{G}$ new radio", IEEE Commun. Standards Mag., vol. 4, no. 3, pp. 22-29, Sept. 2020.

[14] X. Lin et al., "5G New Radio: Unveiling the Essentials of the Next Generation Wireless Access Technology", IEEE Commun. Standards Mag., vol. 3, no. 3, pp. 30-37, Sept. 2019.

[15] 3GPP TS 38.300, NR and NG-RAN Overall Description Stage 2, v16.5.0, Mar. 2021.

Vinay Kumar Shrivastava [SM'20] received his Master's degree in Telecommunications System Engineering from Indian Institute of Technology Kharagpur, India, in 2003 and Bachelor's degree in Electronics Engineering from Rajiv Gandhi Proudyogiki Vishwavidyalaya, India, in 2001. He joined Samsung Electronics in 2003 and has worked extensively on design and development of $3 \mathrm{G} / 4 \mathrm{G} / 5 \mathrm{G}$ modems. He is currently a staff engineer at Samsung Research Institute, Bengaluru, India, and a Samsung 3GPP standardization delegate in RAN2 Working group. His research interests include communications standards, MAC protocols, broadcast communication, device-to-device communications.

Sangkyu Baek [S'06-M'12] received his B.S., M.S. and Ph.D. degrees in mathematics from Korea University, Seoul, Korea, in 2006, 2008, and 2011, respectively. Since 2012, he has been with Samsung Electronics and working on advanced $5 \mathrm{G}$ research and pre-5G standardization. He is currently a staff engineer at Samsung Research, Samsung Electronics, Seoul, Korea, and a Samsung 3GPP standardization delegate in RAN2 Working group. His research interests include communications standards, user plane protocols, URLLC, probability theory, queueing theory, and wireless channel modelling.

Youngkyo Baek received an M.S. degree, in 2002, and finished doctoral course in mathematics from Korea University, Seoul, Korea. He joined Samsung Electronics in 2007 and has worked on 3G/4G/5G standardization especially in WiMAX and 3GPP. He is currently a staff engineer at Samsung Research, Samsung Electronics, Seoul, Korea, and a Samsung 3GPP standardization delegate in SA2 Working group. His research interests include communications standards, non-public network, broadcast communication, proximity services, and queueing theory. 